\title{
LIE PRODUCTS, CLOSED GEODESICS AND FUCHSIAN GROUPS
}

\author{
ALAN F. BEARDON
}

\begin{abstract}
Two results concerning closed geodesics on a Riemann surface are proved. The method of proof indirectly involves the Lie product of two complex matrices.
\end{abstract}

1. Introduction. In this paper we extend the discussion given in [1] in which the Lie product $A B-B A$ of $A$ and $B$ in $\operatorname{SL}(2, C)$ is used to derive a result on geodesics on a Riemann surface. The essential idea in [1] is to relate the Lie product to the geometry of hyperbolic three-space and then consider the given Riemann surface as the quotient of a hyperbolic plane section by an appropriate discrete group of isometries.

We shall relate the Lie product of $A$ and $B$ to the classical factorisation of $A$ and $B$ in terms of other isometries of order two and by doing so we reveal that the Theorem in [1] is merely one of a sequence of similar results.

2. Isometries of the hyperbolic plane. Let $\Delta$ denote the hyperbolic plane with $\rho$ the hyperbolic metric; for the moment, we regard $\Delta$ as being embedded in the extended complex plane and the isometries of $\Delta$ are then Möbius transformations of $z$ or $\bar{z}$. Each conformal isometry is either elliptic, parabolic or hyperbolic.

If $A$ is elliptic, then $A$ has a unique fixed point $w$ in $\Delta$ and $A$ is a hyperbolic rotation of angle $2 \theta$, say, about $w$. Note that $A$ can be expressed as $A=\sigma_{2} \sigma_{1}$ where $\sigma_{j}$ is the reflection in a geodesic $L_{j}$ passing through $w$. Either $L_{1}$ or $L_{2}$ can be chosen arbitrarily; the remaining $L_{j}$ is uniquely determined and $L_{1}$ and $L_{2}$ intersect at $w$ at an angle $\theta$.

If $A$ is hyperbolic, then $A$ fixes two distinct points on the boundary of $\Delta$ and the hyperbolic geodesic $\varrho_{A}$ joining these points is called the axis of $A$. Note that $\mathcal{L}_{A}$ is the unique $A$-invariant geodesic in $\Delta$. The displacement $\rho(z, A z), z \in \Delta$, is minimum when $z$ is on $e_{A}$ and then the displacement is the translation length $T_{A}$ of $A$.

Any hyperbolic $A$ can be expressed as $A=\sigma_{2} \sigma_{1}$ with each $\sigma_{j}$ of order two in two different ways. First, we choose two points $w_{1}$ and $w_{2}$ on $\mathcal{L}_{A}$ with $w_{2}$ halfway between $w_{1}$ and $A w_{1}\left(\right.$ so $\left.\rho\left(w_{1}, w_{2}\right)=\frac{1}{2} T_{A}\right)$. Note that $w_{1}$ or $w_{2}$ can be chosen arbitrarily and the remaining $w_{j}$ is then uniquely determined. The first factorisation of $A$ is $A=\sigma_{2} \sigma_{1}$ where $\sigma_{j}$ is a rotation of order two about $w_{j}$. The second factorisation is obtained by constructing the geodesics $L_{j}$ through $w_{j}$ and orthogonal to $\varrho_{A}$. Then $A=\sigma_{2} \sigma_{1}$ where $\sigma_{j}$ now denotes reflection in $L_{j}$.

Received by the editors July 23, 1981.

1980 Mathematics Subject Classification. Primary 53A35. 
The essential step in [1] is to prove that if $A, B$ and $A B A$ are hyperbolic with axes $\mathcal{L}_{A}, \mathcal{L}_{B}$ meeting at $w$, then $\mathcal{L}_{A B A}$ also passes through $w$. In fact, $A B A$ is necessarily hyperbolic (whether or not $\langle A, B\rangle$ is discrete) and we can give a simpler proof of a more general result.

THEOREM 1. Let $A$ and $B$ be isometries with no common fixed point and let $C=A B A$.

(i) If $A$ and $B$ are hyperbolic with $\mathcal{L}_{A}$ and $\mathcal{L}_{B}$ meeting at $w$ in $\Delta$, then $C$ is hyperbolic and $\mathcal{L}_{C}$ also passes through $w$.

(ii) If $A$ and $B$ are hyperbolic with $\mathcal{L}_{A}$ and $\mathcal{L}_{B}$ disjoint, let $L$ be the unique geodesic orthogonal to $\mathcal{L}_{A}$ and $\mathcal{L}_{B}$. If $C$ is hyperbolic then $\mathcal{L}_{C}$ is also orthogonal to $L$; if $C$ is elliptic or parabolic then the fixed point of $C$ lies on $L$.

(iii) If $A$ and $B$ are elliptic, let $L$ be the geodesic containing the fixed points of $A$ and $B$. If $C$ is hyperbolic then $\mathcal{L}_{C}$ is orthogonal to $L$; if $C$ is elliptic or parabolic then the fixed point of $C$ lies on $L$.

(iv) If one of $A$ and $B$ is hyperbolic and the other is elliptic or parabolic, let $L$ be the geodesic through the elliptic or parabolic fixed point and orthogonal to the hyperbolic axis. If $C$ is hyperbolic then $\mathcal{L}_{C}$ is orthogonal to $L$; if $C$ is elliptic or parabolic then the fixed point of $C$ lies on $L$.

Proof. If $A$ and $B$ are hyperbolic then, as $A$ and $B$ have no common fixed point, their axes either meet in $\Delta$ (as in (i)) or have a common orthogonal (as in (ii)).

We show that in every case we can write

$$
A=\sigma_{1} \sigma_{2}, \quad B=\sigma_{2} \sigma_{3}
$$

where $\sigma_{1}, \sigma_{2}$ and $\sigma_{3}$ are either all rotations of order two or all reflections in geodesics. Then

$$
A B A=\left(\sigma_{1} \sigma_{3} \sigma_{1}\right) \sigma_{2}=\sigma_{4} \sigma_{2}
$$

say. If the $\sigma_{j}(j=1,2,3)$ are rotations of order two then so is $\sigma_{4}$. Note that in this case, if $w_{j}$ is the fixed point of $\sigma_{j}$, then $w_{4} \neq w_{2}$ (because $w_{4}=\sigma_{1}\left(w_{3}\right)$ and so $w_{4}=w_{2}$ implies that $w_{1}, w_{2}, w_{3}$ are collinear which, in turn, implies that $\mathcal{L}_{A}=\mathcal{L}_{B}$ ). It follows that in this case, $A B A$ is hyperbolic with $\mathcal{L}_{A B A}$ passing through the fixed point of $\sigma_{2}$.

If the $\sigma_{j}(j=1,2,3)$ are reflections, then so is $\sigma_{4}$, and $A B A$ is elliptic or parabolic when $L_{4}$ and $L_{2}$ meet ( $L_{j}$ being the line of fixed points of $\sigma_{j}$ ), and it is hyperbolic when $L_{4}$ and $L_{2}$ are disjoint. In the first case, the fixed point of $A B A$ is the point $L_{4} \cap L_{2}$ and this is on $L_{2}$; in the second case $\mathcal{L}_{A B A}$ is orthogonal to $L_{2}$ (and to $L_{4}$ ).

These remarks combined with the following explicit description of the factorisation (1) in each of the cases (i)-(iv) yield the desired result. In case (i), we simply put $w_{2}=w$ and let $\sigma_{2}$ be the rotation of order two about $w_{2}$. The rotations $\sigma_{1}$ and $\sigma_{3}$ are chosen appropriately with fixed points on $\mathcal{L}_{A}$ and $\mathcal{L}_{B}$ respectively and (as already noted) $\mathcal{L}_{A B A}$ passes through $w$. In cases (ii), (iii) and (iv) we simply let $\sigma_{2}$ be the reflection in $L$ and the proof is complete.

3. Lie products. We have deliberately proved Theorem 1 without reference to Lie products; we now relate the proof of Theorem 1 to the concept of a Lie product. First, we briefly review the geometry of hyperbolic space. 
Every Möbius transformation

$$
A: z \mapsto \frac{a z+b}{c z+d}, \quad a d-b c=1,
$$

extends uniquely to act as a Möbius transformation on

$$
H^{3}=\{(z, t): z \in C, t>0\}
$$

and the action is given by identifying $(z, t)$ with the quaternion

$$
\zeta=x+i y+t j+O k
$$

and writing $A: \zeta \mapsto(a \zeta+b)(c \zeta+d)^{-1}$, the computation being within the algebra of quaternions.

If $A$ and $B$ leave a disc $\Delta$ invariant, we can (by conjugation) assume that $A$ and $B$ are in $\operatorname{SL}(2, R)$ and that $\Delta$ is $\{x+i y: y>0\}$. It is equally acceptable (and for our purposes, preferable) to consider $A$ and $B$ as isometries of the model

$$
\Delta^{\prime}=\left\{x+t j: x \in R^{1}, t>0\right\}
$$

(a vertical section of $H^{3}$ ) for $A$ and $B$ act on $\Delta^{\prime}$ in the same way as they act on $\Delta$.

Now any hyperbolic or elliptic $X$ in $\operatorname{SL}(2, C)$ has an axis $\mathcal{H}_{X}$ in $H^{3}$ which is the geodesic in $H^{3}$ joining the fixed points of $X$. If $X$ is hyperbolic and in $\operatorname{SL}(2, R)$ then the axes $\mathcal{L}_{X}$ in $\Delta^{\prime}$ and $\mathcal{K}_{X}$ in $H^{3}$ are the same geodesic. If $X$ is elliptic and in $\operatorname{SL}(2, R)$ then $X$ has a unique fixed point $w$ in $\Delta^{\prime}$ and $\mathcal{H}_{X}$ is the geodesic in $H^{3}$ orthogonal to $\Delta^{\prime}$ and passing through $w$.

Given matrices $A$ and $B$ in $\operatorname{SL}(2, C)$, their Lie product is

$$
\varphi(A, B)=A B-B A \text {. }
$$

We can view this matrix as a Möbius transformation provided that it is nonsingular and this is so precisely when $A$ and $B$ have no common fixed point (which we shall assume). As trace $\varphi(A, B)=0$, we see that $\varphi(A, B)$ is elliptic of order two. Moreover (see [1] $) \varphi(A, B)=\varphi\left(B^{-1}, A\right)=-\varphi(B, A)$ and so

$$
A^{-1} \varphi(A, B) A^{-1}=A^{-1} \varphi\left(B^{-1}, A\right) A^{-1}=\varphi\left(A^{-1}, B^{-1}\right)=\varphi(A, B) .
$$

Thus with $\varphi=\varphi(A, B)$, we have $\varphi A^{-1} \varphi^{-1}=A$ and (similarly) $\varphi B^{-1} \varphi^{-1}=B$. This shows that if $A$ and $B$ are hyperbolic or elliptic, then $\varphi$ interchanges the two fixed points of $A$ and of $B$ and so the axis of $\varphi$ in $H^{3}$ is orthogonal to the axes $\mathcal{H}_{A}$ and $\mathcal{H}_{B}$. In fact, this orthogonal is unique so $\varphi(A, B)$ is actually determined by $\mathcal{K}_{A}$ and $\mathcal{H}_{B}$ alone.

Now assume that $A$ and $B$ are in $\operatorname{SL}(2, R)$ and have no common fixed points. If $A$ and $B$ are hyperbolic with $\mathcal{L}_{A}$ and $\mathcal{L}_{B}$ meeting at $w$, then their Lie product restricted to $\Delta^{\prime}$ is a rotation of $\Delta^{\prime}$ of order two about $w$. If $\mathcal{L}_{A}$ and $\mathcal{E}_{B}$ are disjoint, they have a common orthogonal $L$ in $\Delta^{\prime}$ and $\varphi(A, B)$ is a rotation of $H^{3}$ of order two with axis $L$. In this case, the restriction of $\varphi(A, B)$ to $\Delta^{\prime}$ is precisely the reflection in $L$.

If $A$ and $B$ are elliptic there is a unique geodesic $L$ in $\Delta^{\prime}$ containing the fixed points of $A$ and $B$ in $\Delta^{\prime}$ and this is the axis of $\varphi(A, B)$. Again $\varphi(A, B)$ leaves $\Delta^{\prime}$ invariant and acts on $\Delta^{\prime}$ as a reflection in $L$.

Finally, suppose that one of $A$ and $B$ is hyperbolic with axis $\mathcal{L}$ and that the other is elliptic with fixed point $w$ in $\Delta^{\prime}$. Let $L$ be the geodesic in $\Delta^{\prime}$ through $w$ and 
orthogonal to $L$ : then $L$ is the axis of $\varphi(A, B)$ and $\varphi(A, B)$ acts on $\Delta^{\prime}$ as a reflection in $L$. These observations constitute a proof of the following result.

Theorem 2. Let $A$ and $B$ be in $\operatorname{SL}(2, R)$ and be either hyperbolic or elliptic. Then the Lie product $\varphi(A, B)$ leaves $\Delta^{\prime}$ invariant and its action on $\Delta^{\prime}$ is precisely that of $\sigma_{2}$ described in the proof of Theorem 1.

In fact, it is possible (though probably not useful) to represent all of the $\sigma_{j}$ described earlier in terms of Lie products. Suppose for example, that $A$ and $B$ are hyperbolic with $\mathcal{L}_{A}$ and $\mathcal{L}_{B}$ meeting at $w$. Then $A=\sigma_{1} \sigma_{2}$ and $A$ has a square root $A^{1 / 2}$ (with the same axis but one half of the translation length of $A$ ) and $X=$ $A^{1 / 2} B\left(A^{1 / 2}\right)^{-1}$ is hyperbolic with its axis meeting $\varrho_{A}$ at $w_{1}$. We deduce that $\sigma_{1}=\varphi(A, X)$.

4. Matrix computation. It is possible to prove Theorem 1 simply by computing matrices. For example, to prove (i) we may assume that $\Delta$ is the unit disc and that $w=0$. It is then only necessary to show that the sum of the fixed points of $A B A$ is zero and this is easily established from the coefficients of $A B A$.

5. Geodesics on a Riemann surface. Let $R$ be any Riemann surface with the hyperbolic plane as its universal covering surface. Exactly as in [1] we observe that if two closed geodesics on $R$ intersect at $w$, say, then infinitely many closed geodesics on $R$ pass through $w$. This is Theorem 1(i): note that the remark [1, p. 141] is a direct consequence of our factorisation (1). Theorem 1(ii) also yields a result about geodesics on $R$, namely given two closed disjoint geodesics $L_{1}$ and $L_{2}$ on $R$ then there are infinitely many closed geodesics orthogonal to the unique geodesic $L$ which is itself orthogonal to $L_{1}$ and $L_{2}$. The equivalent statement to the remark [1, p. 141] is that if, in Theorem 1(ii), the isometries $A$ and $B$ are oriented in opposite directions, then $\mathcal{L}_{A B A}$ lies between $\mathcal{L}_{A}$ and $\mathcal{L}_{B}$. Note also that we could take $B$ to be a conjugate of $A$; thus the above interpretation also holds when $L_{1}=L_{2}$. Clearly Theorem 1(iii) and (iv) also have similar interpretations in terms of quotient spaces by Fuchsian groups with elliptic elements.

\section{REFERENCES}

1. T. Jørgensen, Closed geodesics on Riemann surfaces, Proc. Amer. Math. Soc. 72 (1978), 140-142.

Department of Pure Mathematics and Mathematical Statistics, University of Cambridge, 16 Mill lane, Cambridge CB2 1SB, ENgland 